\title{
Incorporation of Styrene into a Model Polyolefin for Enhanced Compatibility with Polyisoprene
}

\author{
Supporting Information
}

\author{
Sravya Jangareddy and Richard A. Register ${ }^{*}$ \\ Department of Chemical and Biological Engineering, Princeton University, \\ Princeton, New Jersey 08544, United States
}

\section{Block and block-random copolymer composition determination}

The composition of the block and block-random precursors can be determined from ${ }^{1} \mathrm{H}$ NMR spectroscopy, from the areas of the olefinic polyisoprene (PI) and polybutadiene (PB) peaks, and the aromatic polystyrene (PS) peaks. The peak assignments ${ }^{1,2}$ are as shown in Figure S1. 


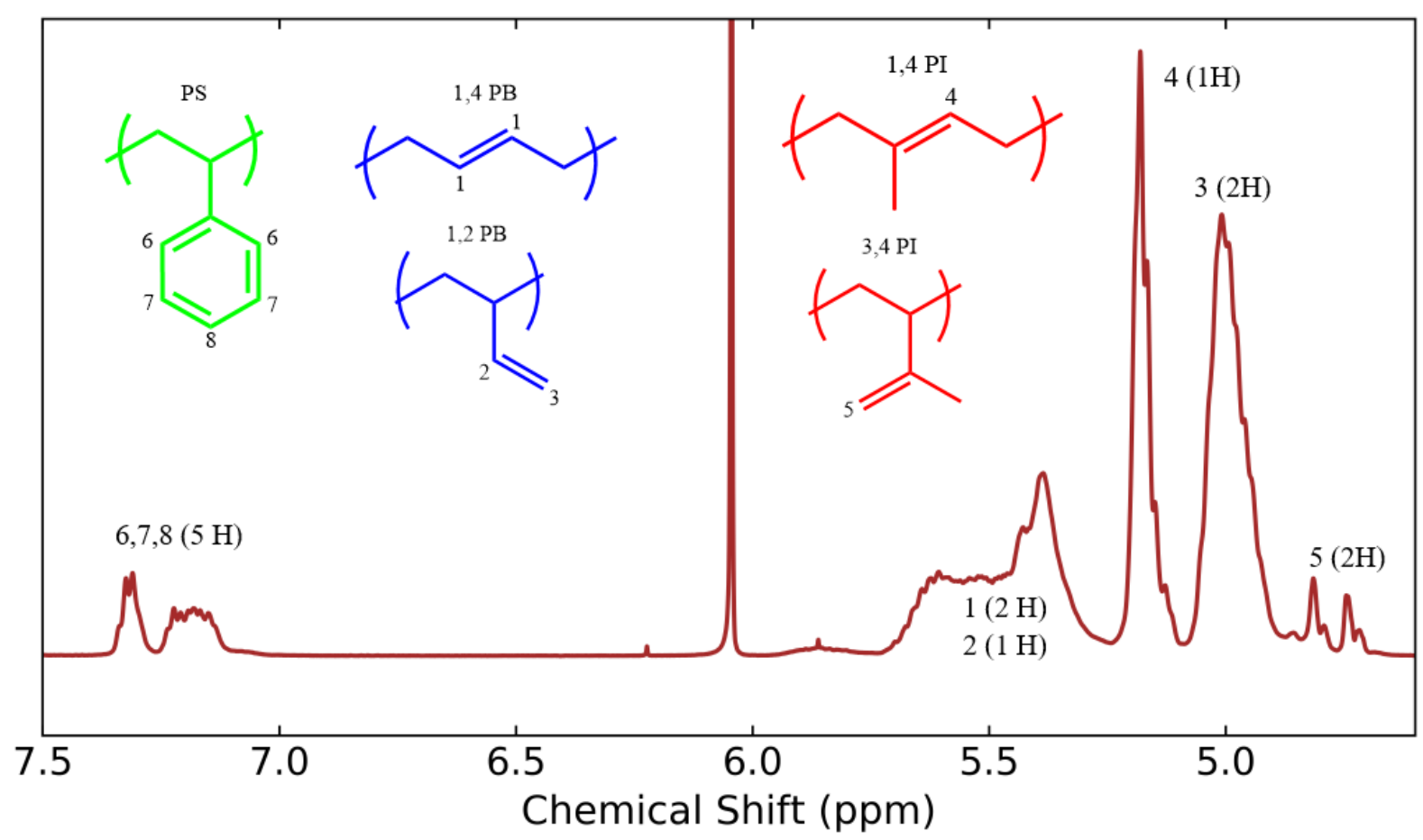

Figure S1. ${ }^{1} \mathrm{H}$ NMR spectrum: olefinic and aromatic peak assignments for PI-SBR10 precursor in 1,1,2,2-tetrachloroethane- $\mathrm{d}_{2}$ (TCE). 


\section{"Hybrid" method for determining levels of saturation/unsaturation of isoprene/butadiene units in block and block-random copolymers}

The homogeneous $\mathrm{Ru}$ catalyst (hRu1) employed for selective saturation of the butadiene units over isoprene and styrene units in the block and block-random copolymers is not perfectly selective. This results in low levels of isoprene saturation and butadiene unsaturation. In the ${ }^{1} \mathrm{H}$ NMR spectrum of the saturated product, the methyl $\left(-\mathrm{CH}_{3}\right)$ peaks from saturated isoprene units (1,4/3,4 addition) and saturated butadiene units (1,2 addition) overlap heavily (0.9 - $0.8 \mathrm{ppm})$. This precludes the determination of low levels of fractional saturation of 1,4 PI directly from the

${ }^{1} \mathrm{H}$ NMR spectrum. Instead, an alternative "hybrid" method that uses both the ${ }^{13} \mathrm{C} N M R$ and ${ }^{1} \mathrm{H}$ NMR spectra was employed to determine the levels of PI saturation/PB unsaturation, as detailed below.

${ }^{13} \mathrm{C}$ NMR: Saturated PI has a $-\mathrm{CH}_{3}$ peak (from 1,4 units) in the aliphatic region at $\sim 19.6 \mathrm{ppm}$ which does not overlap with the unsaturated PI $-\mathrm{CH}_{3}$ peaks, nor the $-\mathrm{CH}_{3}$ peaks from hydrogenated 1,2 PB nor hydrogenated 3,4 PI (Figure S2(a) shows the 1,4 PI $-\mathrm{CH}_{3}$ peak for PIhSBR10-b $\left.\left(T_{O D T}=137^{\circ} \mathrm{C}\right)\right)$. The fractional saturation of 1,4 PI can be determined from the areas of the 1,4 PI $-\mathrm{CH}_{3}$ peak at $~ 19.6 \mathrm{ppm}$ and the 1,4 PI olefinic peak (at $136-134 \mathrm{ppm}$, Figure S2(b)): $8.2 \%$ for PI-hSBR10-b. 

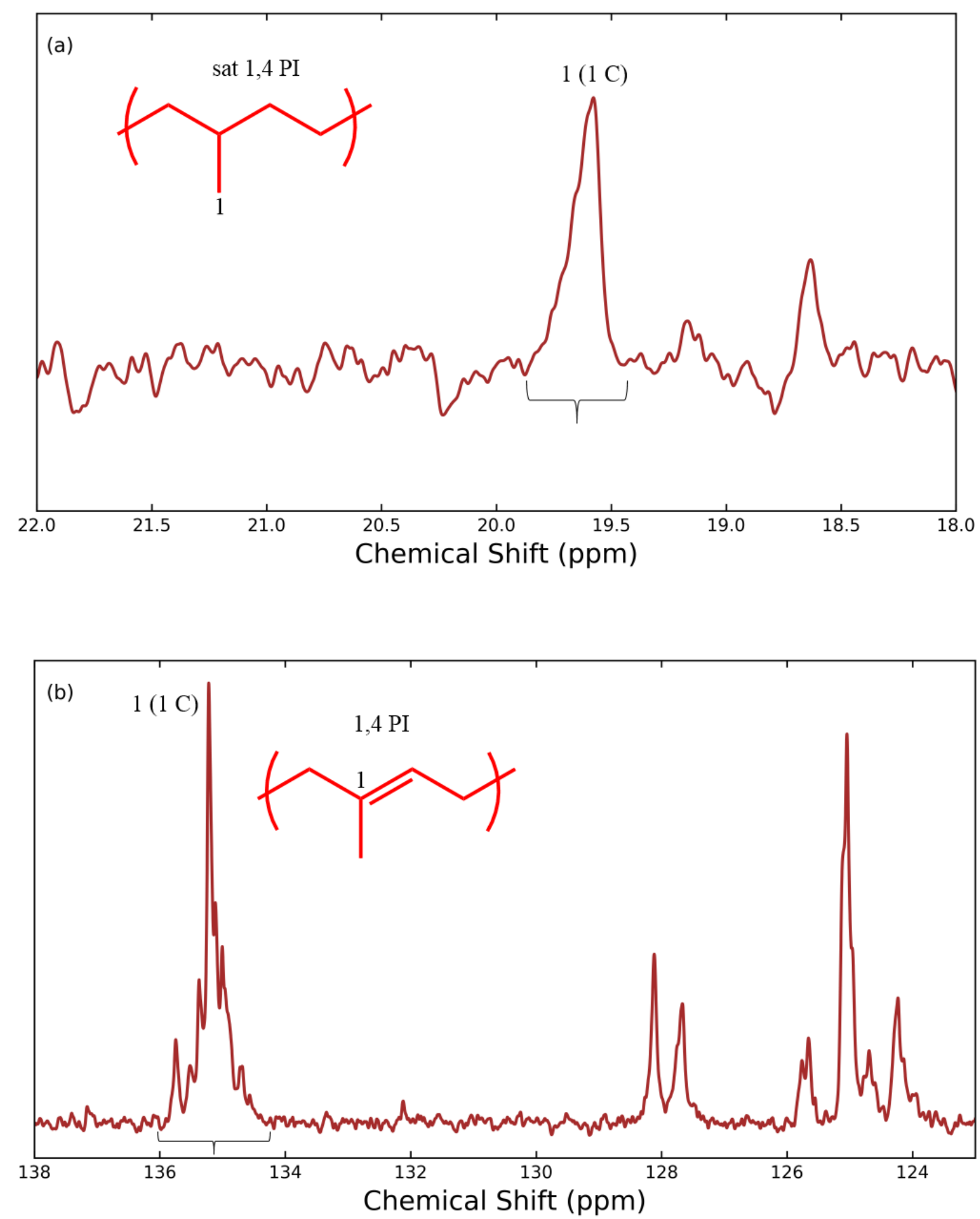

Figure S2. ${ }^{13} \mathrm{C}$ NMR spectra of PI-hSBR10-b in $\mathrm{CDCl}_{3}$ covering the (a) aliphatic and (b) olefinic regions. 
${ }^{1}$ H NMR: The olefinic PI and PB peaks are well-separated in the saturated product (Figure S3). The fractional unsaturation $\left(f_{u}\right)$ of 3,4 PI, 1,4 PB, and 1,2 PB units can be determined from their respective olefinic peak areas with the 1,4 PI olefinic peak as the reference, in combination with the known composition of the precursor polymer and the saturation level of 1,4 PI from the ${ }^{13} \mathrm{C}$ NMR spectrum. Since the fractional unsaturation levels are much less than unity (i.e., 0.69, 0.18 and 0.01 for 3,4 PI, 1,4 PB and 1,2 PB respectively in PI-hSBR10-b), this method produces $f_{u}$ values of sufficient accuracy.

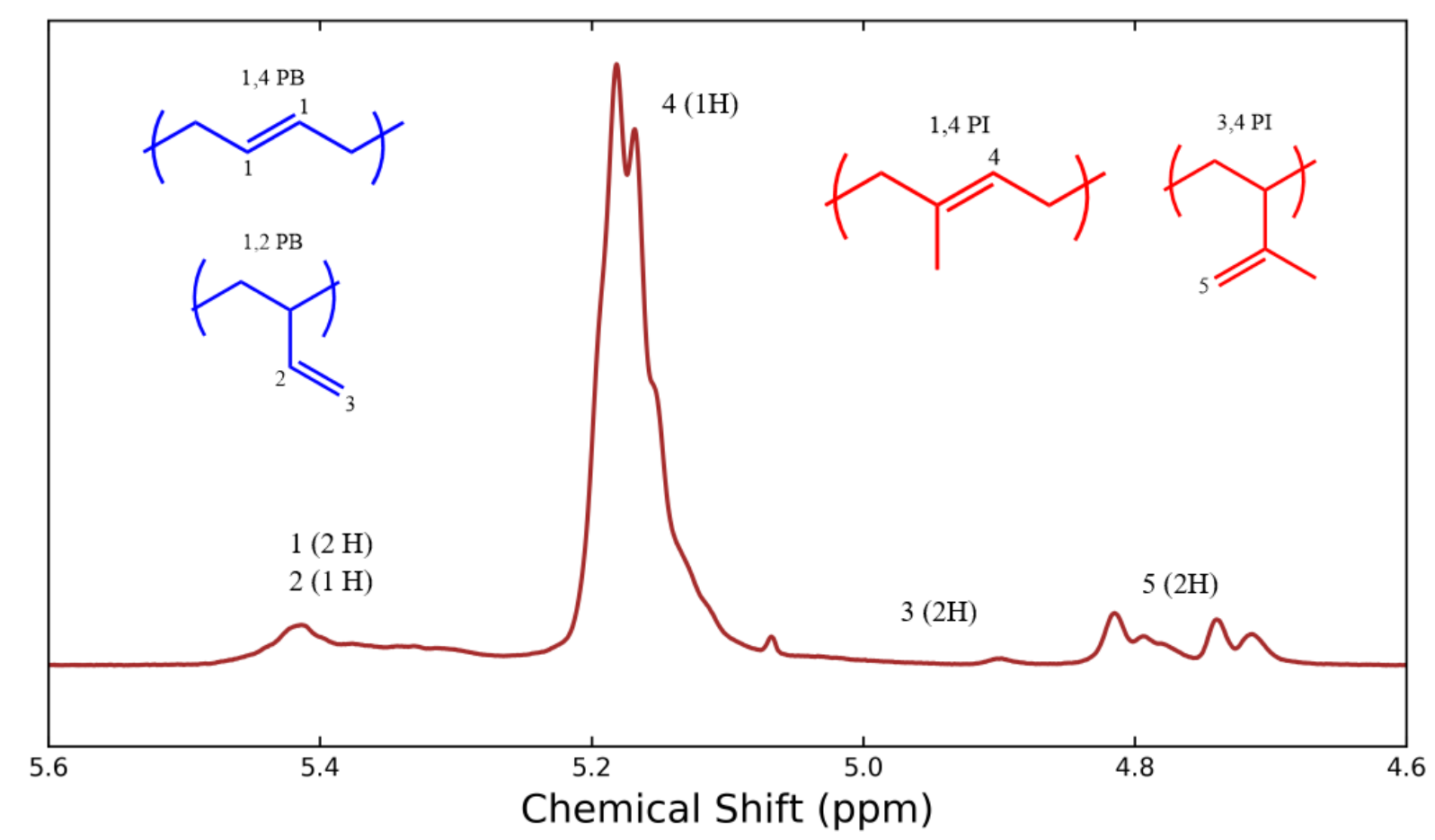

Figure S3. ${ }^{1} \mathrm{H}$ NMR spectrum: olefinic region of PI-hSBR10-b in $\mathrm{CDCl}_{3}$. 


\section{Effect of TMEDA on styrene-butadiene sequence}

We tested the effect of TMEDA (at TMEDA: Li $=4: 1$ ) on a styrene-butadiene copolymerization with $13.3 \mathrm{wt} \%$ styrene in the monomer feed. The polymerization was carried out at $0{ }^{\circ} \mathrm{C}$ to provide ample time for collecting aliquots at different conversions before the polymerization was complete. Reactivity ratios for copolymerization of butadiene (B) and styrene (S) were determined by measuring the integrated polymer compositions at different times (conversions) by ${ }^{1} \mathrm{H} \mathrm{NMR}$, and apparent conversions by GPC (as $M_{n} / M_{n, \text { final }}$, measured as the apparent $M_{n}$ values determined from the elution times calibrated with narrow-distribution polystyrene standards, i.e., "polystyrene-equivalent" values). The instantaneous styrene mole fraction enchained into the copolymer, $F_{S}$, is given by: ${ }^{3}$

$$
F_{S}=\frac{r_{S} f_{S}^{2}+f_{S} f_{B}}{r_{S} f_{S}^{2}+2 f_{S} f_{B}+r_{B} f_{B}^{2}}
$$

With an initial monomer mole fraction of monomer $i, f_{i o}$, the instantaneous polymer composition at different conversions can be determined using Equation $(\mathrm{S} 1) ; r_{S} \cdot r_{B}=1$ was assumed ${ }^{4}$ as the results are insensitive to the value of $r_{S}$ when $f_{S}$ is small. The integrated polymer composition at time step $k$ (mole fraction styrene, $\overline{F_{k}}$ ) can be determined by using:

$$
\overline{F_{k}}=\frac{\sum_{i=0}^{k-1} F_{S, i}\left(p_{i+1}-p_{i}\right)}{p_{k}}
$$

On minimizing the sum of squared residuals at different average compositions, $r_{B}$ can be determined for the styrene-butadiene copolymer as $0.88 \pm 0.04$ ( \pm 1 standard deviation). Since $r_{B}$ $\approx 1$, the composition profile (Figure 1 in the Article) is relatively flat, indicating a nearly random styrene-butadiene sequence. 


\section{Contribution of minor structures to $X_{\text {meas }}$ due to imperfect selectivity of hRu1}

$X_{\text {meas }}\left(T_{O D T}\right)$ can be expressed as a function of $X_{c o r r}\left(T_{O D T}\right)$ and the minor and major structure contributions at $T_{O D T}$ for both PI-hPB and PI-hSBR systems using the multicomponent copolymer equation model as outlined in the Article's section "Effect of Catalyst Selectivity on $X$ " (Equations (5) and (7)). The minor structure contributions were estimated using the $X$ values at $T_{O D T}$, and the known volume fractions $\left(\varphi_{i}\right)$ of these structures within each block (determined from known mole fractions and densities). Table $\mathrm{S} 1$ presents $X(T)$ as a linear function of temperature $\left(X(T)=A+B\left(T-120^{\circ} \mathrm{C}\right)\right)$, calculated from $\chi(T)$ data or expressions in the literature using Equation (2).

Table S1. Temperature parameters describing interaction energies for polymer pairs of interest $\mathrm{t}^{\mathrm{a}}$

\begin{tabular}{|c|c|c|c|c|}
\hline Polymer pair & $A(\mathrm{MPa})$ & $B\left(\mathrm{MPa} /{ }^{\circ} \mathrm{C}\right)$ & $\mathrm{T}$ range $\left({ }^{\circ} \mathrm{C}\right)$ & Reference \\
\hline $\mathrm{I}-\mathrm{hB}$ & 0.72 & $-3.0^{*} 10^{-3}$ & $75-143$ & this work \\
\hline S-I & 3.5 & $-1.2^{*} 10^{-2}$ & $124-267$ & this work, $5^{\mathrm{b}}$ \\
\hline S-hB & 6.4 & $-9.3^{*} 10^{-3}$ & $145-305$ & this work \\
\hline $\mathrm{I}-\mathrm{hI}$ & 1.2 & $-4.6^{*} 10^{-3}$ & $71-101$ & 6 \\
\hline $\mathrm{hI}-\mathrm{B}$ & 1.2 & $-4.6^{*} 10^{-3}$ & $71-101$ & $6^{\mathrm{c}}$ \\
\hline $\mathrm{B}-\mathrm{hB}$ & 0.54 & $-1.0^{*} 10^{-2}$ & $118-166$ & 7 \\
\hline $\mathrm{I}-\mathrm{B}$ & 0.10 & $5.8^{*} 10^{-4}$ & $30-110$ & 8 \\
\hline $\mathrm{hI}-\mathrm{hB}$ & -0.010 & $-2.3^{*} 10^{-5}$ & $50-170$ & 9 \\
\hline $\mathrm{hI}-\mathrm{S}$ & 8.1 & $-1.2^{*} 10^{-2}$ & $121-249$ & $10,11^{\mathrm{d}}$ \\
\hline $\mathrm{B}-\mathrm{S}$ & 1.7 & $-2.2^{*} 10^{-3}$ & $50-180$ & 12 \\
\hline
\end{tabular}

aThough presented here in terms of $X$, these values are easily converted to values of $\chi$ according to the Article's Equation (2), once a value of the molar reference volume $v_{\text {ref }}$ has been chosen. For example, a common choice ${ }^{13}$ is $100 \AA^{3}$ per cell, or $v_{\text {ref }}=60.22 \mathrm{~cm}^{3} / \mathrm{mol}$; such values of $\chi$ are related to $X$ by: $\chi=X v_{\text {ref }} / R T=(X / T)\left(v_{\text {ref }} / R\right)=$ $(X / T)(7.243 \mathrm{~K} / \mathrm{MPa})$, with $T$ in absolute units $(\mathrm{K})$.

${ }^{\mathrm{b}} X_{S-I}(T)$ estimated from block copolymer $X$ determined at $125{ }^{\circ} \mathrm{C}$ in this work (Article's Table 2), and assuming a fractional change in $X$ with $T$ matching that derived from the data in reference 5 , where $X_{S-I}=3.2-$ $0.0010\left(T-120^{\circ} \mathrm{C}\right)$, over the range $124^{\circ} \mathrm{C}<T<267^{\circ} \mathrm{C}$.

${ }^{c} X_{h I-B}$ assumed identical to $X_{I-h I}$ (satisfactory since $\varphi_{h I} \varphi_{B}$ is negligible, $\sim 0.01$ ).

${ }^{\mathrm{d}} X_{h I-S}(T)$ estimated from block copolymer $X$ determined at $126{ }^{\circ} \mathrm{C}$ in reference $11(7.99 \mathrm{MPa})$, and assuming a fractional change in $X$ with $T$ matching that for PS-PEP, derived from the expression in reference 11 based on the data in reference 10 , where $X_{S-E P}=4.8-0.0071\left(T-120^{\circ} \mathrm{C}\right)$, over the range $121^{\circ} \mathrm{C}<T<249{ }^{\circ} \mathrm{C}$. 
Each pair-wise minor structure contribution to $\Delta X$ in PI-hPB systems is calculated as a volume-fraction-weighted $X$, with the weights as assigned by Equation (5) in the Article. The contributions are listed in Table S2(a) and compared graphically in Figure S4(a). In accordance with the equation, $X_{c o r r}\left(T_{O D T}\right)$ (opaque red bar in Figure $\mathrm{S} 4(\mathrm{a})$ ) is obtained by subtracting all the pair-wise contributions (translucent bars) from $X_{\text {meas }}\left(T_{O D T}\right)$ (opaque green bar), and dividing by $\varphi_{I} \varphi_{h B}$

For PI-hSBR systems, each pairwise minor structure contribution to $\Delta X_{2}$ and each pairwise major structure contribution to $\Delta X_{1}$ is calculated as a volume fraction-weighted $X$, with the weights as assigned by Equation (7) in the Article. The contributions are listed in Table S2(b) and compared graphically in Figure S4(b). In accordance with the equation, $X_{c o r r}\left(T_{O D T}\right)$ (opaque red bar in Figure S4(b)) is obtained by subtracting all the pair-wise contributions (translucent bars) from $X_{\text {meas }}\left(T_{O D T}\right)$ (opaque green bar). 
Table S2(a). Pairwise minor structure contributions in PI-hPB diblock copolymers

\begin{tabular}{|c|c|c|c|c|c|c|c|c|c|}
\hline \multirow{2}{*}{ Polymer } & \multirow{2}{*}{$\begin{array}{c}X_{\text {meas }} \text { at } \\
T_{O D T} \\
\text { (MPa) }\end{array}$} & \multicolumn{5}{|c|}{$\begin{array}{l}\text { Pairwise minor structure } \mathrm{X} \\
\text { contributions to } \Delta X(\mathrm{MPa})\end{array}$} & \multirow[t]{2}{*}{$\varphi_{I}$} & \multirow[t]{2}{*}{$\varphi_{h B}$} & \multirow{2}{*}{$\begin{array}{c}X_{\text {corr }} \mathrm{a} \\
T_{O D T} \\
\text { (MPa) }\end{array}$} \\
\hline & & I-hI & B-hB & I-B & B-hI & hI-hB & & & \\
\hline PI-hPB-1a & 0.48 & -0.06 & -0.16 & 0.01 & 0.01 & 0.00 & 0.958 & 0.798 & 0.88 \\
\hline PI-hPB-1b & 0.51 & -0.06 & -0.05 & 0.01 & 0.00 & 0.00 & 0.948 & 0.915 & 0.71 \\
\hline PI-hPB-2a & 0.49 & -0.06 & -0.05 & 0.01 & 0.01 & 0.00 & 0.946 & 0.868 & 0.71 \\
\hline PI-hPB-2b & 0.50 & -0.05 & -0.02 & 0.01 & 0.00 & 0.00 & 0.947 & 0.917 & 0.65 \\
\hline
\end{tabular}

Table S2(b). Pairwise minor and major structure contributions in PI-hSBR block-random copolymers

\begin{tabular}{|c|c|c|c|c|c|c|c|c|c|c|c|c|}
\hline \multirow[t]{2}{*}{ Polymer } & \multirow{2}{*}{$\begin{array}{c}X_{\text {meas }} \\
\text { at } \\
T_{O D T} \\
\text { (MPa) }\end{array}$} & \multicolumn{7}{|c|}{$\begin{array}{l}\text { Pairwise minor structure } \mathrm{X} \text { contributions to } \Delta X_{2} \\
\text { (MPa) }\end{array}$} & \multicolumn{3}{|c|}{$\begin{array}{l}\text { Pairwise major structure } \mathrm{X} \\
\text { contributions to } \Delta X_{1}(\mathrm{MPa})\end{array}$} & \multirow{2}{*}{$\begin{array}{c}X_{\text {corr }} \\
\text { at } \\
T_{O D T} \\
\text { (MPa) }\end{array}$} \\
\hline & & S-hI & I-hI & B-hB & S-B & I-B & B-hI & hI-hB & $\mathrm{I}-\mathrm{hB}$ & S-hB & S-I & \\
\hline PI-hSBR10-a & 0.31 & 0.03 & -0.06 & -0.04 & -0.01 & 0.01 & 0.01 & 0.00 & -0.10 & 0.05 & -0.01 & 0.43 \\
\hline PI-hSBR10-b & 0.32 & 0.07 & -0.10 & -0.02 & -0.01 & 0.01 & 0.01 & 0.00 & -0.10 & 0.03 & -0.03 & 0.46 \\
\hline PI-hSBR20 & 0.23 & 0.09 & -0.09 & -0.04 & -0.02 & 0.01 & 0.01 & 0.00 & -0.10 & 0.07 & -0.04 & 0.35 \\
\hline PI-hSBR30 & $<0.20$ & 0.13 & -0.09 & -0.05 & -0.03 & 0.01 & 0.01 & 0.00 & -0.10 & 0.11 & -0.06 & $<0.28$ \\
\hline PI-hSBR60 & 0.40 & 0.12 & -0.03 & 0.00 & -0.04 & 0.01 & 0.00 & 0.00 & -0.04 & 0.15 & -0.04 & 0.27 \\
\hline
\end{tabular}



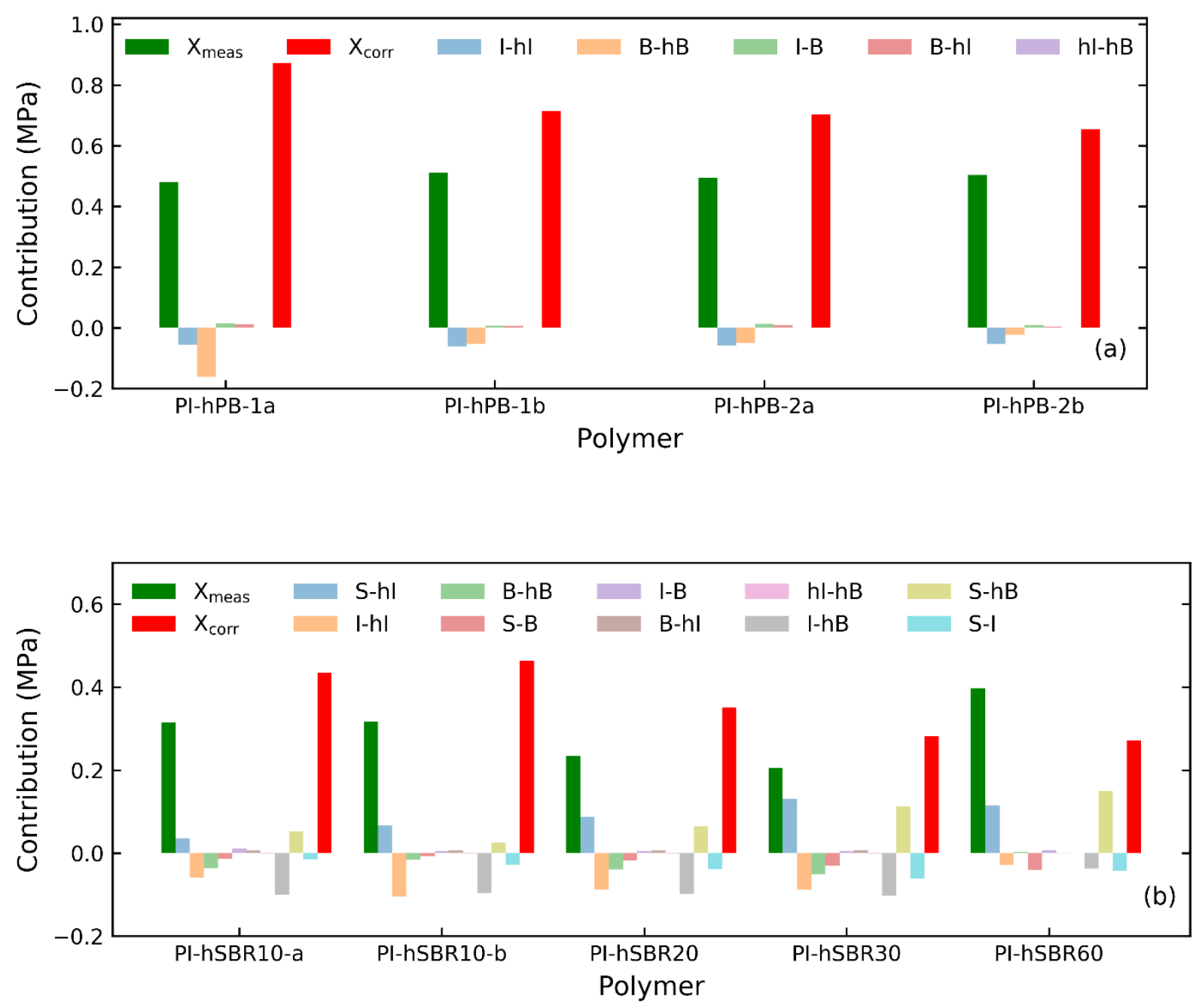

Figure S4. $X_{\text {corr }}, X_{\text {meas }}$, and $X$ contributions (a) from minor structures to $\Delta X$ due to imperfect selectivity in PI-hPB, and (b) from major structures to $\Delta X_{1}$ and minor structures to $\Delta X_{2}$ in PI-hSBR systems. 


\section{Uncertainty estimates for $X_{\text {meas }}$ and $X_{c o r r}$}

By far the largest source of random error in $X_{\text {meas }}$, as per the Article's Equation (2), is the uncertainty in $M_{n}$ from GPC, which we estimate as $\pm 5 \%$ ( \pm 1 standard deviation), considering the uncertainty in $d n / d c$, and the influence of baseline and integration window selection on both $M_{w}$ and $\boxplus$. By contrast, the uncertainty in $\rho$ is less than $0.2 \%$, and the uncertainty in $T_{O D T}\left( \pm 2{ }^{\circ} \mathrm{C}\right)$ is less than $0.6 \%$. We do not consider any possible systematic error in the values of $(\chi N)_{O D T}$ calculated via Equation (3); the random errors (through the uncertainty in $\bar{N}$, via the uncertainty in $M_{n}$ and $\left\langle R^{2}\right\rangle_{0} / M$, as per Equation (4)) are again much smaller than the direct effect of the uncertainty in $M_{n}$.

In calculating $X_{\text {corr }}$ from $X_{\text {meas }}$ via Equation (5) or (7), we consider only the additional contributions from the uncertainties in the fractions of undesired minor structures (B and hI), which are calculated assuming a $\pm 1 \%$ error in all the NMR peak area integrations used in the "hybrid" method of composition determination. No contribution is included from random error in the various $X(T)$ functions (Table $\mathrm{S} 1$ ) used for the correction, or any systematic error in the applicability of the copolymer equation, from which Equations (5) and (7) are derived.

\section{Can hPB be treated as a homopolymer in the copolymer equation model (CEM)?}

In PI-hPB and PI-hSBR, the hydrogenated butadiene (hB) units are a mixture of $1,4 \mathrm{hB}$ and $1,2 \mathrm{hB}$ structures. In calculating the interaction energy densities via the CEM, we treated $\mathrm{hPB}$ as a homopolymer with an averaged monomeric unit (denoted BV below). In this "averaged" case, the CEM expression for $X$ is given by: 


$$
X_{I-B V r S}=\varphi_{B V} X_{I-B V}+\varphi_{S} X_{I-S}-\varphi_{B V} \varphi_{S} X_{S-B V}
$$

where $\varphi_{B V}=\varphi_{B}+\varphi_{V}=1-\varphi_{S}$.

Instead, if $\mathrm{hPB}$ is treated as a random copolymer of $\mathrm{B}$ and $\mathrm{V}$, the CEM expression for $X$ can be written as:

$$
X_{I-B r V r S}=\varphi_{B} X_{I-B}+\varphi_{V} X_{I-V}+\varphi_{S} X_{I-S}-\varphi_{B} \varphi_{S} X_{B-S}-\varphi_{V} \varphi_{S} X_{V-S}-\varphi_{V} \varphi_{B} X_{B-V}
$$

To satisfy $X_{I-B V r S}=X_{I-B r V r S}$, by comparing Equations (S3) and (S4), the "effective" $X_{I-B V}$ and $X_{S-B V}$ must correspond to the groupings below:

$$
\begin{aligned}
& X_{I-B V}=f_{B} X_{I-B}+f_{V} X_{I-V}-f_{B} f_{V} X_{B-V} \\
& X_{S-B V}=f_{B} X_{S-B}+f_{V} X_{S-V}-f_{B} f_{V} X_{B-V}
\end{aligned}
$$

where $f_{V}=\varphi_{V} /\left(\varphi_{B}+\varphi_{V}\right)$ and $f_{B}=1-f_{V}$.

Equations (S5-a) and (S5-b) imply that hPB can be treated as a homopolymer with an averaged monomeric unit in predicting the interaction energies, as in Equation (S3), as long as $f_{V}$ (vinyl content of $\mathrm{hPB}$ ) remains essentially constant at all levels of styrene incorporation. Note that a similar situation pertains for the PI block, which consists of three distinct units (cis, trans, and $3,4)$, where the ratio of these three is essentially constant across all the polymers studied.

However, Table 1 in the Article shows that $f_{V}$ decreases from $\sim 0.76$ to $\sim 0.50$ on going from $0 \mathrm{wt} \% \mathrm{~S}$ to $\sim 53 \mathrm{wt} \% \mathrm{~S}$, a trend also observed by Halasa et al. previously. ${ }^{14}$ So, some assessment of how strongly $X_{S-h B}$ varies with $f_{V}$ is warranted. The value of $X_{S-h B}=5.7 \mathrm{MPa}$ (at $200{ }^{\circ} \mathrm{C}$ ) at $f_{V}=0.81$ determined in this work (Table S1) may be compared with $X_{S-h B}=6.5 \mathrm{MPa}\left(\right.$ at $200{ }^{\circ} \mathrm{C}$ ) determined from the $T_{O D T}$ of a PS-hPB diblock copolymer with $f_{V}=0.08 .{ }^{15}$ The similarity in $X$ values despite the large $(\sim 0.73)$ difference in $f_{V}$ suggests a weak dependence of $X_{S-h B}$ on 
microstructure, and that $X_{S-h B}$ may be adequately treated as independent of $f_{V}$ for the modest range in $f_{V}(\sim 0.26)$ relevant here. No literature data are available to evaluate the dependence of $X_{I-h B}$ on $f_{V}$, but we hypothesize a similarly modest dependence; note that $X_{I-h B}$ is approximately fourfold smaller than $X_{S-h B}$ in any case.

It may be worthwhile to note that the aforementioned comparison indicates that $X_{S-h B}$ decreases with $f_{V}$. This is contrary to the expectation of regular mixing, given that $\delta_{P S}>$ $\delta_{h P B}$, and that $\delta_{h P B}$ is known to decrease with increasing $f_{V} .{ }^{16}$ This indicates that the threecomponent system of S, B, and V (where B and V represent hydrogenated 1,4 and 1,2-butadiene units, respectively) does not obey regular mixing, further complicating an assessment of how $X_{S-h B}$ might depend on hPB microstructure. 


\section{References:}

(1) Tanaka, Y.; Takeuchi, Y.; Kobayashi, M.; Tadokoro, H. Characterization of Diene Polymers. I. Infrared and NMR Studies: Nonadditive Behavior of Characteristic Infrared Bands. $J$. Polym. Sci. Part A-2 Polym. Phys. 1971, 9, 43-57.

(2) Bovey, F. A.; Hood, F. P.; Anderson, E. W.; Snyder, L. C. Polymer NMR Spectroscopy. XI. Polystyrene and Polystyrene Model Compounds. J. Chem. Phys. 1965, 42, 3900-3910.

(3) Alfrey, T.; Goldfinger, G. The Mechanism of Copolymerization. J. Chem. Phys. 1944, 12, 205-209.

(4) Beckingham, B. S.; Sanoja, G. E.; Lynd, N. A. Simple and Accurate Determination of Reactivity Ratios Using a Nonterminal Model of Chain Copolymerization. Macromolecules $\mathbf{2 0 1 5}, 48,6922-6930$.

(5) Khandpur, A. K.; Förster, S.; Bates, F. S.; Hamley, I. W.; Ryan, A. J.; Bras, W.; Almdal, K.; Mortensen, K. Polyisoprene-Polystyrene Diblock Copolymer Phase Diagram near the OrderDisorder Transition. Macromolecules 1995, 28, 8796-8806.

(6) Bates, F. S.; Rosedale, J. H.; Stepanek, P.; Lodge, T. P.; Wiltzius, P.; Fredrickson, G. H.; Hjelm, R. P. Static and Dynamic Crossover in a Critical Polymer Mixture. Phys. Rev. Lett. 1990, 65, 1893-1896.

(7) Qiu, J.; Mongcopa, K. I.; Han, R.; López-Barrón, C. R.; Robertson, M. L.; Krishnamoorti, R. Thermodynamic Interactions in a Model Polydiene/Polyolefin Blend Based on 1,2Polybutadiene. Macromolecules 2018, 51, 3107-3115.

(8) Hasegawa, H.; Sakurai, S.; Takenaka, M.; Hashimoto, T.; Han, C. C. Small-Angle Neutron Scattering and Light Scattering Studies on the Miscibility of Protonated Polyisoprene/Deuterated Polybutadiene Blends. Macromolecules 1991, 24, 1813-1819. 
(9) Graessley, W. W.; Krishnamoorti, R.; Reichart, G. C.; Balsara, N. P.; Fetters, L. J.; Lohse, D. J. Regular and Irregular Mixing in Blends of Saturated Hydrocarbon Polymers. Macromolecules 1995, 28, 1260-1270.

(10) Lai, C. Block Copolymer Phase Behavior: From Melt to Solution. Ph.D. Thesis, Princeton University, 1999.

(11) Beckingham, B. S.; Register, R. A. Synthesis and Phase Behavior of Block-Random Copolymers of Styrene and Hydrogenated Isoprene. Macromolecules 2011, 44, 4313-4319.

(12) Owens, J. N.; Gancarz, I. S.; Koberstein, J. T.; Russell, T. P. Investigation of the Microphase Separation Transition in Low-Molecular-Weight Diblock Copolymers. Macromolecules 1989, 22, 3380-3387.

(13) Eitouni, H. B.; Balsara, N. P. Thermodynamics of Polymer Blends. In Physical Properties of Polymers Handbook; Mark, J. E., Ed.; Springer: New York, NY, 2007; pp 339-356.

(14) Chang, C. C.; Halasa, A. F.; Miller, J. W.; Hsu, W. L. Modelling Studies of the Controlled Anionic Copolymerization of Butadiene and Styrene. Polym. Int. 1994, 33, 151-159.

(15) Beckingham, B. S.; Burns, A. B.; Register, R. A. Mixing Thermodynamics of Ternary BlockRandom Copolymers Containing a Polyethylene Block. Macromolecules 2013, 46, 27602766.

(16) Graessley, W. W.; Krishnamoorti, R.; Balsara, N. P.; Butera, R. J.; Fetters, L. J.; Lohse, D. J.; Schulz, D. N.; Sissano, J. A. Thermodynamics of Mixing for Blends of Model EthyleneButene Copolymers. Macromolecules 1994, 27, 3896-3901. 